“(C) 2021 IEEE. Personal use of this material is permitted. Permission from IEEE must be obtained for all other uses, in any current or future media, including reprinting/republishing this material for advertising or promotional purposes, creating new collective works, for resale or redistribution to servers or lists, or reuse of any copyrighted component of this work in other works." 


\title{
A Platform for Real-Time Space Health Analytics as a Service Utilizing Space Data Relays
}

\author{
Carolyn McGregor \\ Ontario Tech University \\ Simcoe St North, Oshawa, Canada \\ University of Technology Sydney \\ Ultimo, Sydney, Australia \\ c.mcgregor@ieee.org
}

\begin{abstract}
The health, wellness and adaptation response of astronauts during spaceflight is a key component for the success of any manned mission. Physiological and psychological responses of astronauts during spaceflight have been monitored from the first manned missions sixty years ago. However, limited communication networks to and within the spacecraft have limited methods to monitor the health, wellness and adaptation response of astronauts in real-time. This has resulted in a paradigm of astronaut monitoring as discontinuous samplings of physiological data that are captured on board the spacecraft and transported to Earth on storage devices for retrospective down sampled analysis. In 2009, as part of prior research, McGregor proposed a big data analytics framework and platform, that enables the capture and processing of physiological data and other clinical data in real-time for new approaches to real-time health monitoring. The platform, was named the Artemis platform after the Greek goddess of childbearing as the first domain it was used was neonatal intensive care. Its efficacy and reliability as a new approach for real-time health monitoring has been demonstrated in the critical care domain and specifically within the domain of neonatal intensive care. McGregor previously proposed the application of Artemis as an approach for autonomous health monitoring within the spacecraft to support missions within and beyond low Earth orbit. This would enable sophisticated realtime health, wellness and adaptation assessment that did not require the transmission of data beyond the spacecraft. Artemis Cloud has been proposed as a cloud-based approach to provide remote health monitoring. Artemis Cloud enables Health Analytics as a Service and has been demonstrated utilizing the Ontario Research and Innovation Optical Network (ORION) in Ontario and Artemis Cloud instances located at the Compute Ontario advanced research computing node within the Centre for Advanced Computing, Queen's University, Ontario providing remote health monitoring of neonatal intensive care patients at two hospitals in Ontario, Canada. There is great potential for this Health Analytics as a Service model enabled through Artemis Cloud to support the assessment of health, wellness and adaptation response of astronauts in space, however, robust and reliable networks are required. The next stage of space exploration will see unmanned missions using robots that will require prognostics and health management. This will be followed by manned lunar orbiters, Moon bases and Mars missions that will all require the support of health, wellness and adaptation assessment of astronauts engaged in those missions. Deep space hybrid radio frequency and optical networks have great potential to address the current gap in space communication networks. This paper presents a framework and infrastructure to enable real-time equipment monitoring for prognostics and health management and astronaut health monitoring through cloud-based Health
\end{abstract}

Analytics as a Service utilizing space data relays. A key benefit of this approach is its ability to monitor their health and wellbeing onboard the spacecraft as well as enabling the equipment and astronaut's physiological data, and other clinical data, to be sent to an Earth based Mission Control Center within more manageable latencies of seconds or minutes. This will provide a more viable alternative to autonomous only approaches for equipment and astronaut monitoring.

\section{TABLE OF CONTENTS}

1. INTRODUCTION .1

2. ASTRONAUT HEALTH .........................................2

3. DATA COLLECTION FOR HEALTH MONITORING AND EQUIPMENT PHM IN SPACE .3

4. ONLINE HEALTH ANALYTICS DURING

SPACEFLIGHT 4

5. TRANSMISSION OF HEALTH DATA TO EARTH ......6 6. Commstar Space data Relay as a Service (SDRAAS) .6

7. A FRAMEWORK FOR REAL-TIME SPACEFLIGHT HeAlTh ANALYTICS AS A SERVICE UTILIZING SDRAAS .8

8. A Platform FOR REAL-Time SPACE HeALTH ANALYTICS AS A SERVICE UTILIZING SDRAAS .....10 9. CONCLUSION AND FUTURE WORK .....................11 ACKNOWLEDGEMENTS ......................................11

REFERENCES ........................................................... 12

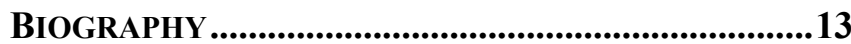

\section{INTRODUCTION}

Humanity has a desire to explore space beyond Low Earth Orbit (LEO), with initiatives to enable lunar orbit, Moon and Mars exploration set to rise in the years to come. Space exploration is an extreme environment and impacts human physiology and psychology. The physical distances from Earth pose challenges for responsiveness to medical emergencies. To date the research on adaptation has been limited to understanding adaptation in LEO. Adaptstion behaviors in cislunar and on the Moon surface are still unknown. As a result, the need to monitor astronauts pre, during and post space missions to assess the health, wellness 
and adaptation response during spaceflight is a key component for the success of any manned mission.

Collection of health data from astronauts has occurred from the first space flight [1]. However, limited communication networks to and within the spacecraft have limited methods to monitor the health, wellness and adaptation response of astronauts in real-time. This has resulted in a paradigm of astronaut monitoring as discontinuous samplings of physiological data that are captured on board the spacecraft and transported to Earth on storage devices for retrospective down sampled analysis. In recent years, the options for data transfer have included radio frequency connection between the Earth and the International Space Station (ISS) but this data is down sampled from the full dataset and monitoring devices are still worn discontinuously [1].

Monitoring astronauts within the spacecraft, during transportation to the spacecraft and during extra vehicular activities (EVAs) is a Big Data challenge. In addition, the real-time monitoring of the spacecraft and the equipment within the spacecraft is a Big Data challenge. Similar challenges will exist within cislunar space, on the Moon and ultimately on Mars.

Big Data frameworks and platforms have been proposed on Earth for new approaches to real-time health monitoring. McGregor has previously proposed the Artemis platform as an artificial intelligence Big Data based clinical decision support system platform to monitor preterm infants [2], [3]. McGregor further demonstrated the application of this approach for astronaut monitoring in space [4]. The focus of that research was to demonstrate how Artemis could be used to support autonomous health monitoring within the spacecraft with data transfer to Earth when the opportunity to do so was available. At the time, it was believed that on a mission to Mars there would be weeks at a time when there would be no communication with Earth. Due to the limitations of data transmission to Earth, the paradigm for future research directions to provision health monitoring in cislunar and deep space broadly has been to create spaceflight clinical decision support system solutions that have a high level of autonomy and self-sufficiency.

The provision of Health Analytics as a Service via Artemis Cloud through a cloud based version of Artemis has been demonstrated [5]-[7]. Addressing the current limitations of space data transmission approaches and doing so with suitable bandwidth will enable paradigm changing approaches for the provision of human health monitoring and equipment prognostic and health monitoring in space and in particular, beyond LEO, as a service from Earth.

CommStar Space Communications and Laser Light Communications have proposed a new form of space data relay provisioned as a service for space (SDRaaS) [8]. This new data relay infrastructure that supports radio frequency and optical has great potential to address the current limitations for deep space communications both in terms of signal latency and bandwidth. As a result, new approaches for the provision of health analytics for those in space from Earth becomes possible.

This paper presents a framework to enable real-time astronaut health monitoring and equipment monitoring for prognostics and health management through cloud-based Health Analytics as a Service utilizing SDRaaS. The framework is instantiated within a platform utilizing SDRaaS provisioned by Commstar and Laser Light.

Section 2 outlines the impact of space as an extreme environment on astronaut health and wellness and the opportunities of high frequency monitoring. Section 3 outlines the data collection methods for health monitoring in space together with a brief introduction to prognostic and health management of equipment in space. Section 4 explores recent research in the domain of online health analytics during space flight. In section 5, the approaches for the delivery of health data to Earth is presented. Section 6 introduces CommStar and Laser Light and their proposed approach for a space data relay between cislunar, the Moon and the Earth. In section 7, a framework for real-time spaceflight health analytics as a service utilizing (SDRaaS) is presented. That framework is instantiated in a platform in section 8 where the hardware and or software for each component is introduced. The paper concludes in section 9 with a discussion of future work.

\section{Astronaut Health}

Space is an extreme environment for humans and, as a result, the ongoing monitoring of astronaut health, wellness and adaptation is required through any mission. Weightlessness or microgravity and confinement gives rise to a range of different physiological and psychological responses. Physiologically, space impacts the musculoskeletal system, the cardiovascular system, the neurosensory system, the immune system and creates metabolic changes. It causes several psychological effects due to physical isolation, confinement, a lack of privacy and restricted or enforced interpersonal contacts. Astronauts are exposed to higher levels of radiation than on Earth, are at risk of solar flares, and cabin toxicity [9]. In addition, they are susceptible to terrestrial pathophysiologies and disease.

As a result, regular assessment of astronaut health, wellness and adaptation response is required. These regular astronaut health assessments follow terrestrial guidelines and are based on differential diagnosis and currently known pathophysiologies of disease from decades of human physiology research using data captured from prior missions. Astronauts are observed during a space mission discontinuously through periodic health checks, examinations and checklists [10]. However, high frequency physiological monitoring performed in terrestrial intensive care populations is demonstrating that new previously unknown pathophysiologies are evident in high speed physiological data monitored continuously [3], [11]. In 
addition, emergency situations can arise and would benefit from continuous physiological monitoring [10].

Beyond traditional health checks, Baevsky proposed the functional state algorithm [12] that analyses discontinuous electrocardiogram segments for Russian cosmonauts and provides an assessment of wellness and adaptation. However, the software created to perform that analysis is located on Earth and the analysis is performed retrospectively post mission [1], [13].

As work progresses to take humans beyond LEO it will be critical to use new real-time approaches to understand the adaptation process in Cislunar, on the surface of the Moon and ultimately for the journey to and on the surface of Mars.

Real-time health monitoring in space using high frequency physiological data has great potential to improve health, wellness and adaptation in space.

\section{DATA COLLECTION FOR HEALTH MONITORING AND EQUIPMENT PHM IN SPACE}

\section{Health Monitoring within the Spacecraft}

The first successful manned spaceflight was completed by Yuri Gagarin when he travelled into space on the Vostok 3KA-3 spacecraft on 12th April 1961. During that mission electrocardiogram data was captured. Upon his return to Earth, the electrocardiogram was analysed to assess overall heart rhythm function as well as RR peak distance from paper traces of his electrocardiogram readings to assess the impact of launch, orbit and return on cardiac function and in turn on the human body in general.

NASA Astronauts on the Project Mercury wore a harness to monitor electrocardiogram (ECG), blood pressure, respiration rate and body temperature [14].

In recent years the Russian cosmonauts have utilized the Cosmocard ECG Holter monitor style device on the ISS and within various analogue missions within the NEK isolation (Mars 500) facility [13] and their Dry Immersion tanks [15] to collect cosmonaut ECG data.

The Bio-Monitor (also known as the Astroskin) was launched for use on the ISS as part of a SpaceX Dragon cargo spaceflight on Dec $4^{\text {th }}, 2018$ [16]. First worn by Canadian Astronaut David Saint-Jacques, it will be commissioned until 2023. It consists a smart shirt and dedicated tablet application. The Bio-Monitor is a variant of the Hexoskin for use in space. The Bio-Monitor measures: electrocardiogram (ECG) at 256Hz; heart rate as derived from the ECG; respiration at $128 \mathrm{~Hz}$; breathing rate as derived from respiration; tidal volume as derived from respiration; systolic blood pressure; skin temperature; peripheral blood oxygen saturation; full-body orthogonal triaxial acceleration at $64 \mathrm{~Hz}$; and physical activity levels as derived from the full-body orthogonal triaxial acceleration. Data is collected within a data logger/battery pack that is attached to the shirt that can be disconnected and connected to a base station to extract the data [17] [18].

\section{Health Monitoring During EVAs}

An important aspect of many space missions is to perform various maintenance activities and other tasks and experiments outside the spacecraft. These activities require the astronauts to wear extra-vehicle (EVA) suits.

As part of International Space Station Phase 1 (or ShuttleMir) missions, that occurred between March 1995 and May 1998, the NASA astronauts wore the Space Shuttle Extravehicular Mobility Unit (EMU). During testing, the participants wore ECG leads to enable the collection of electrocardiogram data for the derivation of a heart rate. Heart rate and rhythm data together with carbon dioxide concentration and other suit parameters were provided to ground via telemetry every two minutes. EMU terrestrial testing mostly occurred underwater in the neutral buoyancy laboratory (NBL) at NASA's Johnson Space Center in Houston, Texas. The Russian cosmonauts wore a $5^{\text {th }}$ generation Orlan suit. [19].

The VPack biomedical monitoring system was proposed to support continuous physiological monitoring of crew in space for extra-vehicle (EVA suit) and planetary surface activities. Data is collected, acquired, integrated, analysed and archived by NASA's communication, avionic and informatics (CAI) computational subsystem on the outside of the space suit via 2 RS232 serial connections [9], [20]. Astronaut privacy is ensured as data collection is in accordance with the Health Insurance Portability and Accountability Act (HIPAA). Plethysmograph, skin temperature and skin galvanic are sampled at $100 \mathrm{~Hz}$ for all but the pulse oximetry which was captured as an analog signal that was converted to a 5 byte signal at a rate of $75 \mathrm{~Hz}$. Data acquisition is enabled via a low power personal area network enabled through a non-invasive wireless sensor mesh network. VPack provides data integration and performs analysis of physiological vital signs, activity level, fall detection and location to detect and predict deviations from expectations. VPack integrates with the CAI via a Visual Basic program running on Windows within the CAI that receives data packets at a rate of $5 \mathrm{~Hz}$ and archives the data within a database within the CAI. Remote access to the VPack software was demonstrated to be possible from a simulation habitat using the wireless mesh network and VNC software. As a result, vital signs were provided to the subject in the suit via a head mounted display within the helmet of the suit and in the remote location to ensure that the subject was not strained. One interesting finding was that communication consumes more energy than computation. VPack was tested at the Johnson Space Centre, Houston, Texas, USA and in a simulated MARS environment in Flagstaff, Arizona.

\section{Spacecraft, Equipment and Structure PHM}

To enable prognostics and health management of space vehicles, hardware and software technologies are embedded 
in the vehicle subsystems, launch and mission operations, and maintenance operations. This enables the construction of a digital twin of the vehicle that can provide both real-time and life-cycle vehicle health information. An integrated vehicle health management (IVHM) system has been developed and implemented to monitor environmental health of vehicles during orbit flight [20].

For habitats and other structures on the Moon and Mars, approaches used for terrestrial smart buildings can be applied. Within smart buildings both the construction and operational life of the building can be monitored through the integration of sensors during construction and the use of either construction and operation based building information models (BIM) [21]. This has key application of space structures to monitor the building during construction and then while it is in operation. As a result, there is great potential to utilize "as a service" approaches for spacecraft, equipment and structure PHM.

\section{Online Health AnAlytics during SPACEFLIGHT}

In prior work McGregor proposed a Big Data driven artificial intelligence based online health analytics platform, Artemis, named after the Greek goddess of childbearing, to provide a new approach for clinical decision support. [22]. In that initial deployment, the Artemis Platform was located on computing equipment within the hospital. It has been and continues to be demonstrated within the context of its use in neonatal intensive care units (NICUs) that provide care for premature and ill term infants [2], [3], [7].

McGregor proposed Artemis as a platform for real-time online health analytics during spaceflight in [23]. In that work she demonstrated the potential of Artemis for autonomous astronaut monitoring on the ISS and long range missions. The architectural model, as shown in Figure 1, proposes that the data acquisition, data collection, online analytics, results presentation, data persistency and knowledge discovery components are located on the spacecraft.

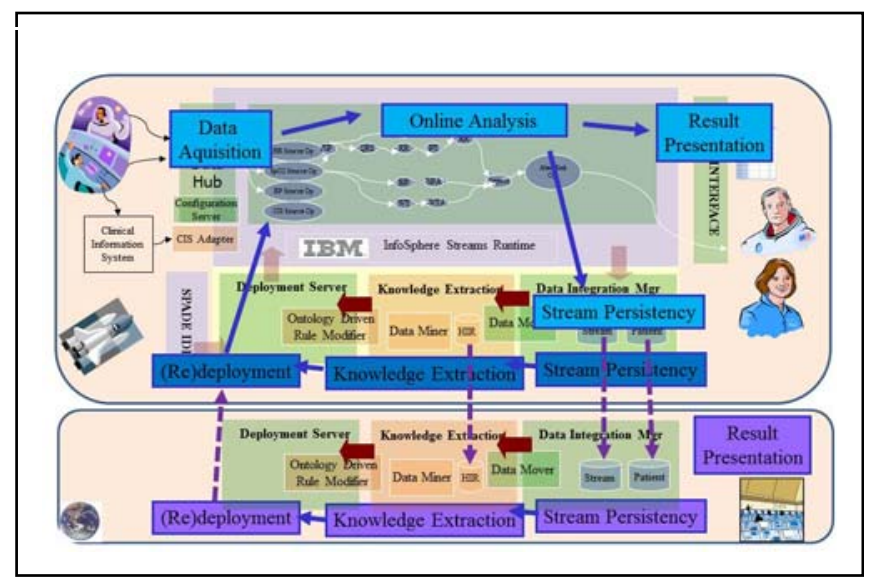

Figure 1. Artemis for Space [4]
In addition, at times where network traffic and network connectivity allow, raw data and the results of analytics performed on the spacecraft are downloaded to the Earth based Artemis companion deployment that contains data persistency, online analytics, results presentation and knowledge discovery components. This Earth based component has the benefit of greater computing power and can be utilized by more human and AI resources. For the spacecraft component however, the extent of online analytics updates and knowledge discovery performed on the data are limited to computing and human resources available during the space flight.

Artemis was extended utilizing cloud computing to propose Artemis Cloud as a Health Analytics as a Service approach for clinical decision support [5]. This was initially demonstrated via a case study where a set of lower frequency physiological data sampled at a reading a second $(1 \mathrm{~Hz})$ from the SpaceLabs physiological monitors within the NICU, Women and Infants Hospital, Providence, Rhode Island, where transmitted within HL7 data packets to an instance of Artemis Cloud at the University of Ontario Institute of Technology (now known as Ontario Tech University).

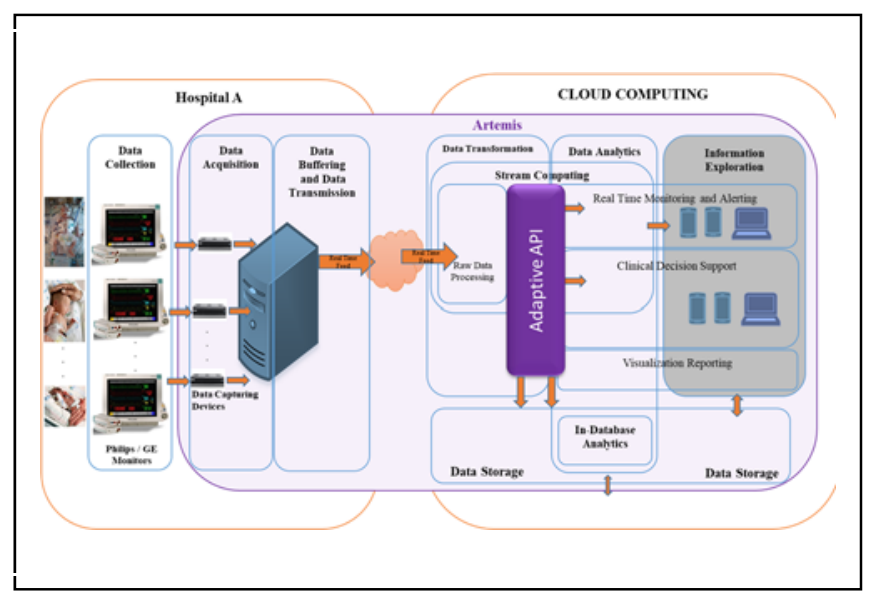

Figure 2. Artemis Cloud for HAaaS [6]

Artemis Cloud was further extended and deployed in a case study connecting to all 48 Level 3 NICU McMaster Children's Hospital bed spaces along with the 3 delivery suite neonatal bed spaces and all 17 bed spaces at the NICU, Southlake Regional Health Centre which is a Level 2 NICU. In that work the two Artemis Cloud instances, one for each hospital, were located at the Centre for Advanced Computing (CAC), Queen's University's which operates as one of the Advanced Research Computing nodes within Compute Ontario. Within these deployments data was transmitted to the Artemis instances at the CAC through secure VPN within the Ontario Research and Innovation Optical Network (ORION). The VPN for the McMaster connection was 1GB and the VPN for the Southlake connection was 100MB [6], [7]. A diagram depicting the Artemis Cloud platform these deployments is shown in Figure 2. 
Yeung and McGregor proposed an extended Artemis Cloud for use on the ISS as shown in Figure 3. In this version, both physiological data from the astronauts and countermeasure device data is captured and integrated within the same platform. This enables assessment of the short term and longer term impact of the use of the countermeasure devices [24]. While this framework enabled the decoupling of data acquisition from the remaining components and included the ability to buffer and transmit data, the computing components were still on board the same spacecraft, namely the ISS.

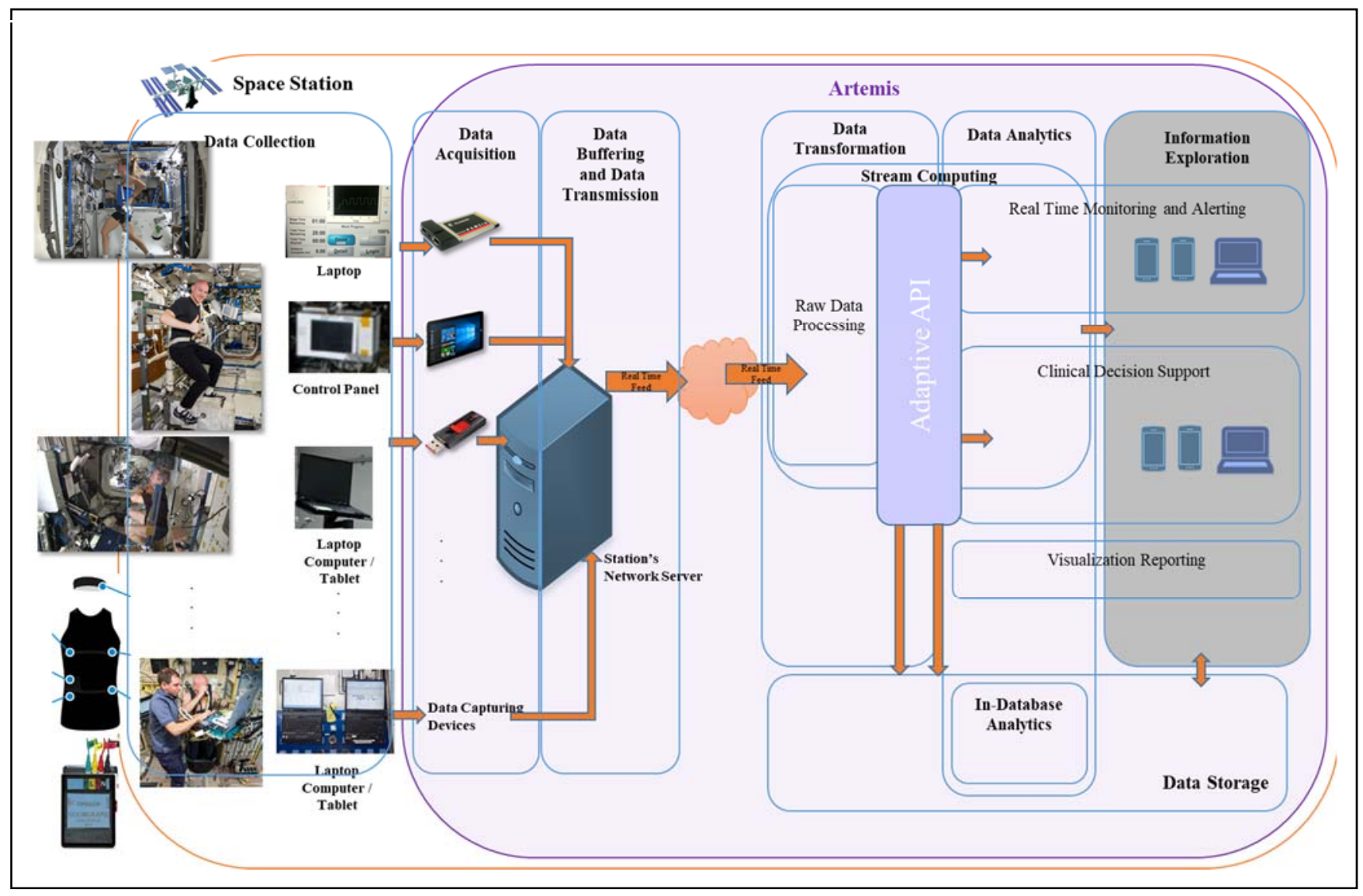

Figure 3. Proposed Framework for Spaceflight integrating Countermeasure Data (ACMS) [24]

The Advanced Crew Medical System (ACMS) was previously proposed by the Canadian Space Agency [25]. A conceptual diagram of an ACMS for the provision of Space Medicine Decision Support System is presented in Figure 4.

The goal of the ACMS is to provide on board autonomous health assessment to assist the Chief Medical Officer (CMO) onboard the spacecraft with health assessment of all the crew. The inputs to the ACMS are the Pre-flight medical history, In-flight non-medical data, medical devices data, clinical observations and personal assessments. The Data Processing and Handling Component processes all data received for the Output component to create information and knowledge. The Output component enables the generation of: astronaut health status, diagnosis and medical telemetry and treatment recommendations including countermeasures. It also supports data archiving for research and the management of medical consumables.
The ACMS conceptual diagram proposes that all data is forwarded to an Electronic Medical Record database. It does not support real-time analysis of streaming physiological data together with other sensor based data for real-time health monitoring. It does contain important operational components relevant for space that are not contained in terrestrial health monitoring systems.

Despite decades of technological research and development, the capacity of medical decision support system aboard the space station is still very limited. A significant amount of analytics is performed retrospectively, upon return to Earth [1], necessitating further research to support the growing spaceflight medical care demands, especially as humans embark on missions of longer distance and duration. Substantial effort is still required to build a knowledge base for use on board the spacecraft. 


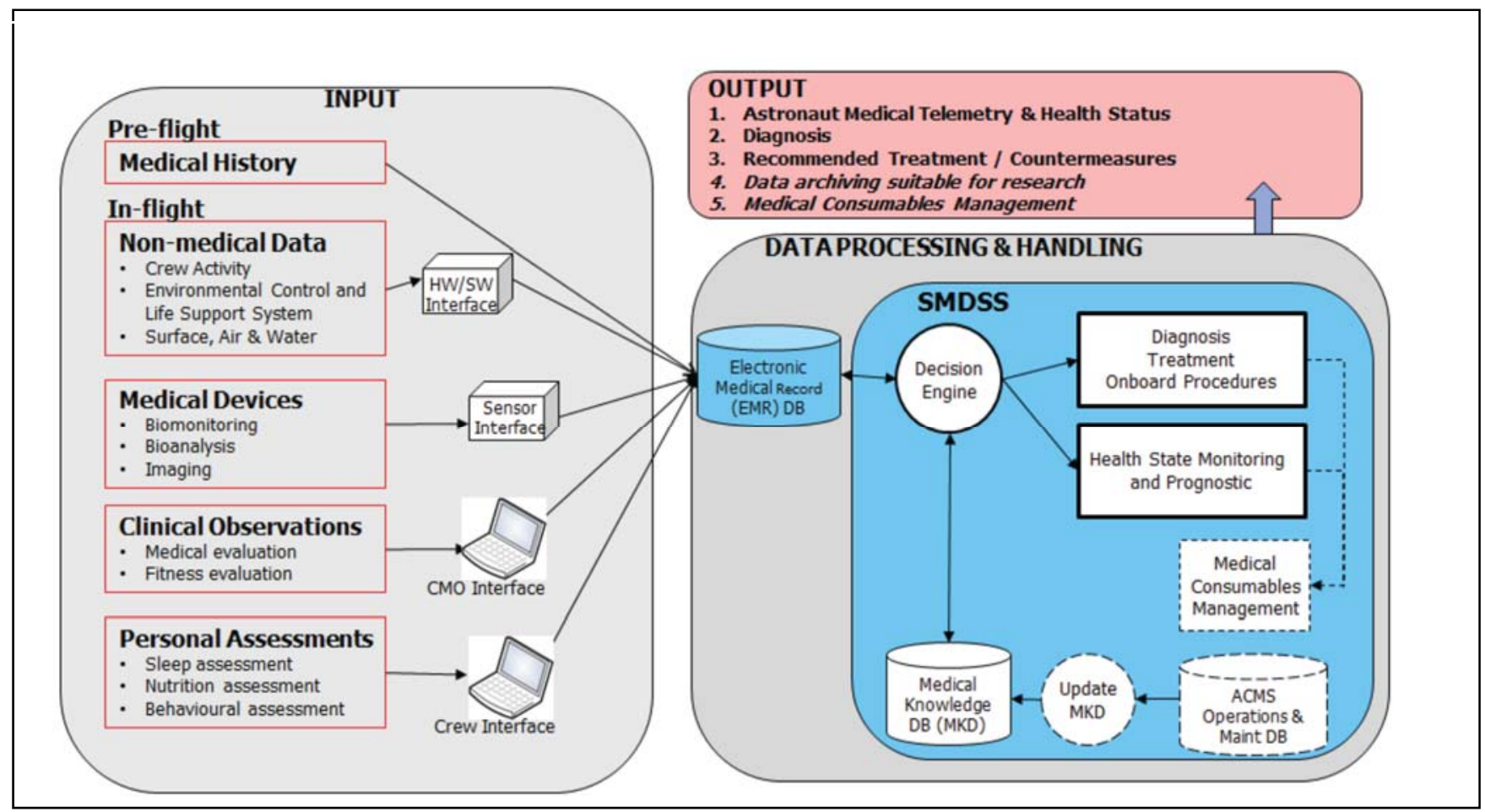

Figure 4. Conceptual Diagram of an Advanced Crew Medical System (ACMS) [25]

\section{Transmission of Health Data to Earth}

In the early years of space flight, health data was collected during the mission and returned to Earth post mission for retrospective analysis.

Currently, near continuous, bi-directional communications with launch vehicles, the ISS and the Hubble Space Telescope is provisioned by NASA through radio frequency (RF) via a system of Tracking and Data Relay Satellites (TDRS) and ground based antennas within the Deep Space Network. (DSN) [26]. The TDRS are in a high orbit above Earth and strategically placed to enable the transmission from anywhere in orbit. The ground-based antenna's utilize landlines to send the data to various NASA centers. In 2019 this was upgraded including an upgrade of the software-based modem on the ISS. Currently there is a $600 \mathrm{Mbps}$ connection between the ISS and NASA on Earth. Transmission latency is in just a few milliseconds [27].

As noted previously, the Artemis Cloud deployment at McMaster Children's hospital utilized a 1GB VPN connection. As a result, future planning for Cislunar and Luna habitat bandwidth to support the transmission of health data will require increased capacity than that currently available for communication with the ISS to change the paradigm from autonomous health monitoring beyond LEO to the provision of Space Health Analytics as a Service (SHAaaS) from Earth.

In addition, there will be unmanned missions beyond LEO for deployment of equipment and other supplies before manned missions beyond LEO occur. As a result, for equipment that is deployed and commissioned, prognostics and health monitoring (PHM) of that equipment will be required.

\section{Commstar Space Data Relay as a SERVICE (SDRAAS)}

On June $16^{\text {th }}$, 2020, Commstar Space Communications, www.comstar.space, declared its intention to deploy a nextgeneration proprietary hybrid data relay satellite ("CommStar-1") between the Earth and the Moon by 2023 [8]. This will enable a paradigm shift from taxpayer-funded communications to the use commercial infrastructures. Commstar-1 will be situated 41,632 miles from the Moon and will operate as an advanced network access point in the Cislunar service area by providing both optical and radio frequency communications across the 225,000 miles between the Moon and Earth through the provision of Space Data Relay as a Service (SDRaaS) [8]. 


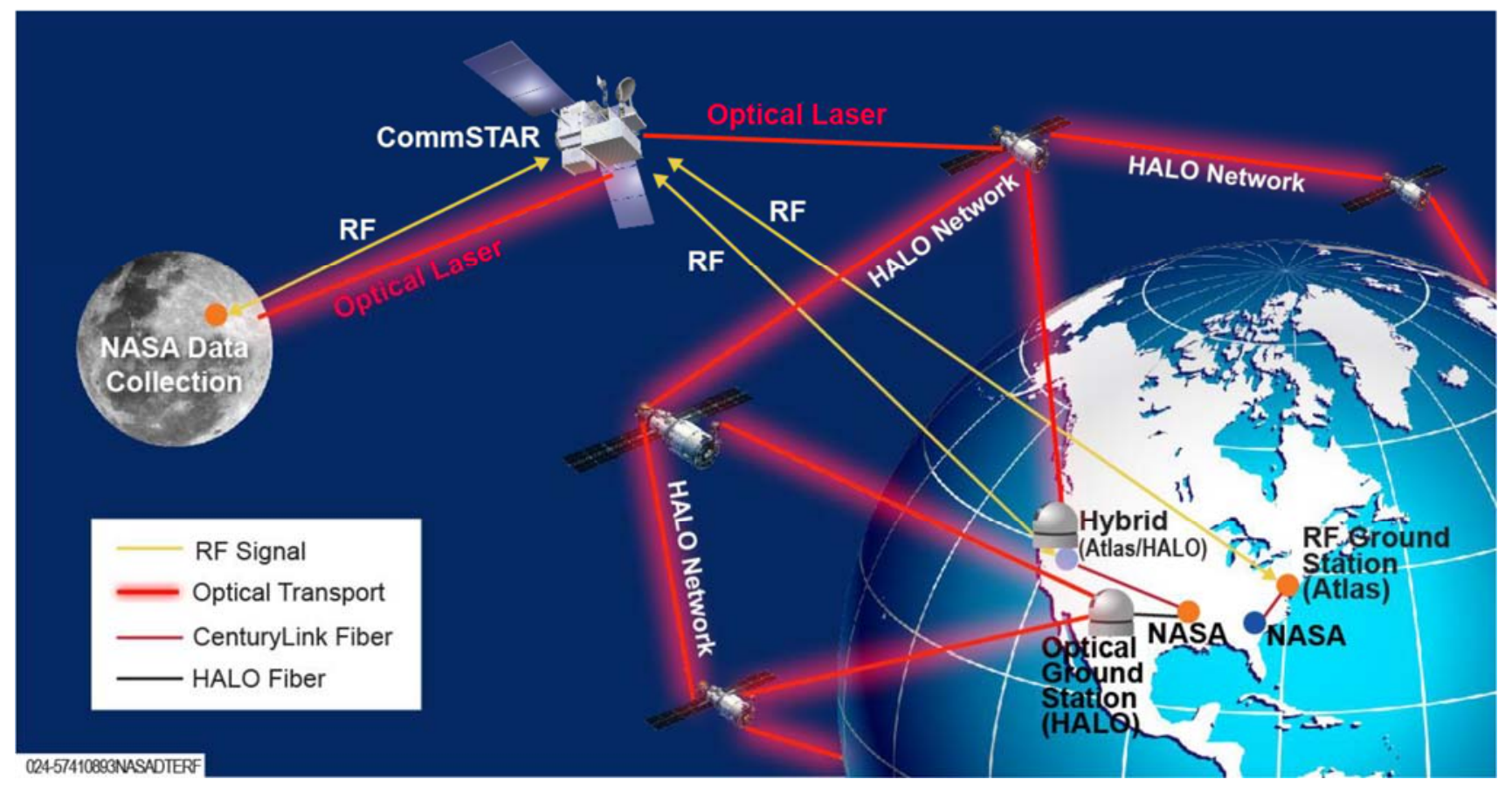

Figure 5. CommStar Architecture [28]

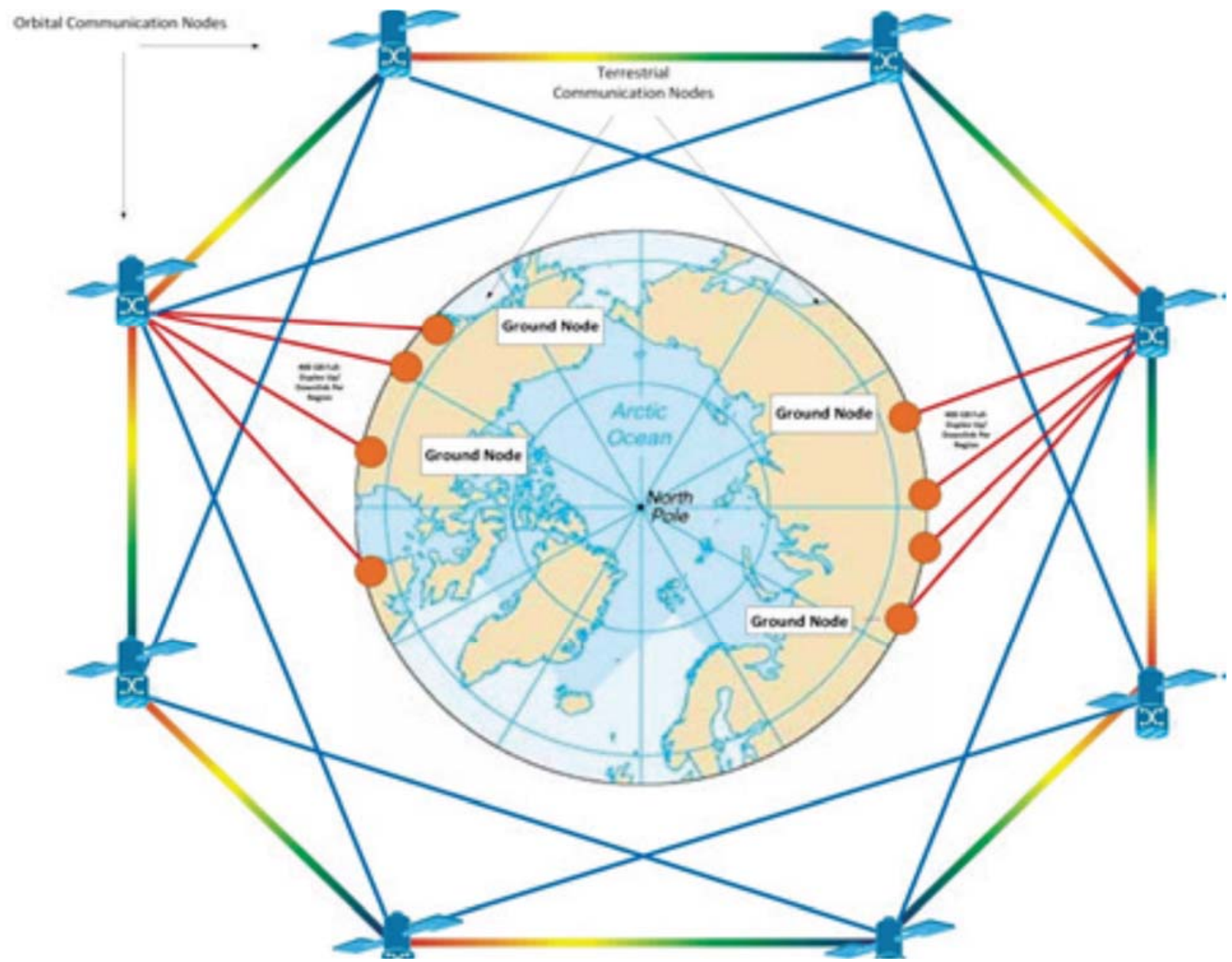

Figure 6. HALO Network Architecture showing eight HALO satellites [29] 
To enable the communications to Earth, CommStar will transmit radio frequency communications directly to $\mathrm{RF}$ antenna ground stations on the Earth, or will transmit optical transmissions via the HALO Network as shown in Figure 5. The HALO network contains a constellation of 12 Medium Earth Orbit satellites with a service capacity of "33Tbps comprised of 48 satellite to satellite (sat-sat) 200Gbps optical crosslinks and 72 satellite to ground (sat-ground) 100Gbps optical bidirectional links" [30]. This satellite network will connect to the Ground Node Segment (GNS) consisting of the StarEdge ground based receive and transmit stations, which will enable the connection with customer Points of Presence (PoPs) [31].

This proposed SDRaaS creates significant opportunities to propose Earth hosted cloud-based prognostics and health management systems along with human health, wellness, resilience and adaptation monitoring for those in space utilizing Earth hosted Space Health Analytics as a Service.

\section{A FRAMEWORK FOR REAL-TIME SPACEFLIGHT HEALTH ANALYTICS AS A SERVICE UTILIZING SDRAAS}

In this section a framework for real-time Space Health Analytics as a Service (SHAaaS) is proposed that utilizes SDRaaS. It builds on McGregor's prior research that proposed an extension to her Artemis platform, created to provide real-time clinical decision support for neonates, to propose a platform for health monitoring in space [4]. It further extends her Artemis Cloud platform, that provisions Health Analytics as a Service [5], [6] to propose a framework for real-time SHAaaS.

The framework for SHAaaS is presented in Figure 7. The SHAaaS framework contains the big data analytics based approaches required for the real-time analysis of Internet of Things (IoT) based devices namely: data collection, data acquisition, data transformation, data analytics, data storage, knowledge discovery, data visualisation and visual analytics. It supports real-time and retrospective knowledge discovery. Examples of Data Collection components currently used on the ISS together with components of the SDRaaS provisioned through CommStar and the HALO Network are also included in Figure 7 to provide details of the platform instantiation that are outlined further in Section 8.

Management of IoT data within the location of the data acquisition IoT devices, is known as edge computing (EC) [32], facilitating efficient data processing in real-time with minimal or no latency. The SHAaaS framework supports EC within the concept of cloud computing enabling the data collection and data acquisitions components to exist within the Edge Computing component and supported by Data Buffering and Data Transmission to enable the delivery of that data to the Data Transformation component in another location.
The framework is flexible and accommodates different configurations from predominantly autonomous to predominantly utilizing the SRAaaS. The predominantly autonomous configuration is demonstrated by the Cislunar habitat within Figure 7. Within this configuration, all components are present to support a degree of autonomy. In addition, data can be forwarded to another location from the Data Buffering and Data Transmission component as well as the Data Storage component. In this way. Additional realtime and retrospective processing of the data can occur at another location, on Earth for example, for further exploration of the data for operational purposes or research. The second configurational option is an EC configuration with the EC component shown behind the cislunar habitat in Figure 7. Within this option, Data Collection, Data Acquisition along with Data Buffering and Data Transmission is enabled at the edge. Some preprocessing of data is also possible within the Data Buffering and Data Transmission component to support EC. Data is then transmitted through the SDRaaS to an instance on Earth that contains the remaining architectural components operating as a remote SHAaaS and denoted in the diagram by the one or more Artemis instances. In the diagram two instances are denoted, one operated in Canada by Compute Ontario and the other in Australia by the Pawsey high performance computing facility. The SDRaaS may relay the data via PoP locations for the Canadian Space Agency and Australian space agency before it is routed to Compute Canada and Pawsey respectively.

Finally, the third option outlined is a full service model where only Data Collection occurs at the location. In this context the SDRaaS connects directly to the device to relay the data to the SHAaaS. The SDRaaS performs the role of Data Acquisition and utilizes the SDRaaS Data Buffering and Transmission elements to manage the transfer of the data to the SHAaaS instances on Earth or other location.

Some of the instantiated platform components for different architectural components within the SHAaaS framework such as examples of data collection devices currently within the habitat and the components of the CommStar Data Space Relay are presented in Figure 7 as examples and also discussed further in section 8 .

Currently functional requirements such as health consumable management are outside the scope of this framework. However, as stock management can be seen as a stream computing problem, tracking consumables as they are used, its inclusion in the future is possible.

This approach also can serve as a foundation for the provision of prognostics and health management of equipment in space through Space PHM as a Service (SPHMaaS) and this is introduced at the end of this section.

The components of the SHAaaS framework are detailed further in the following subsections. 


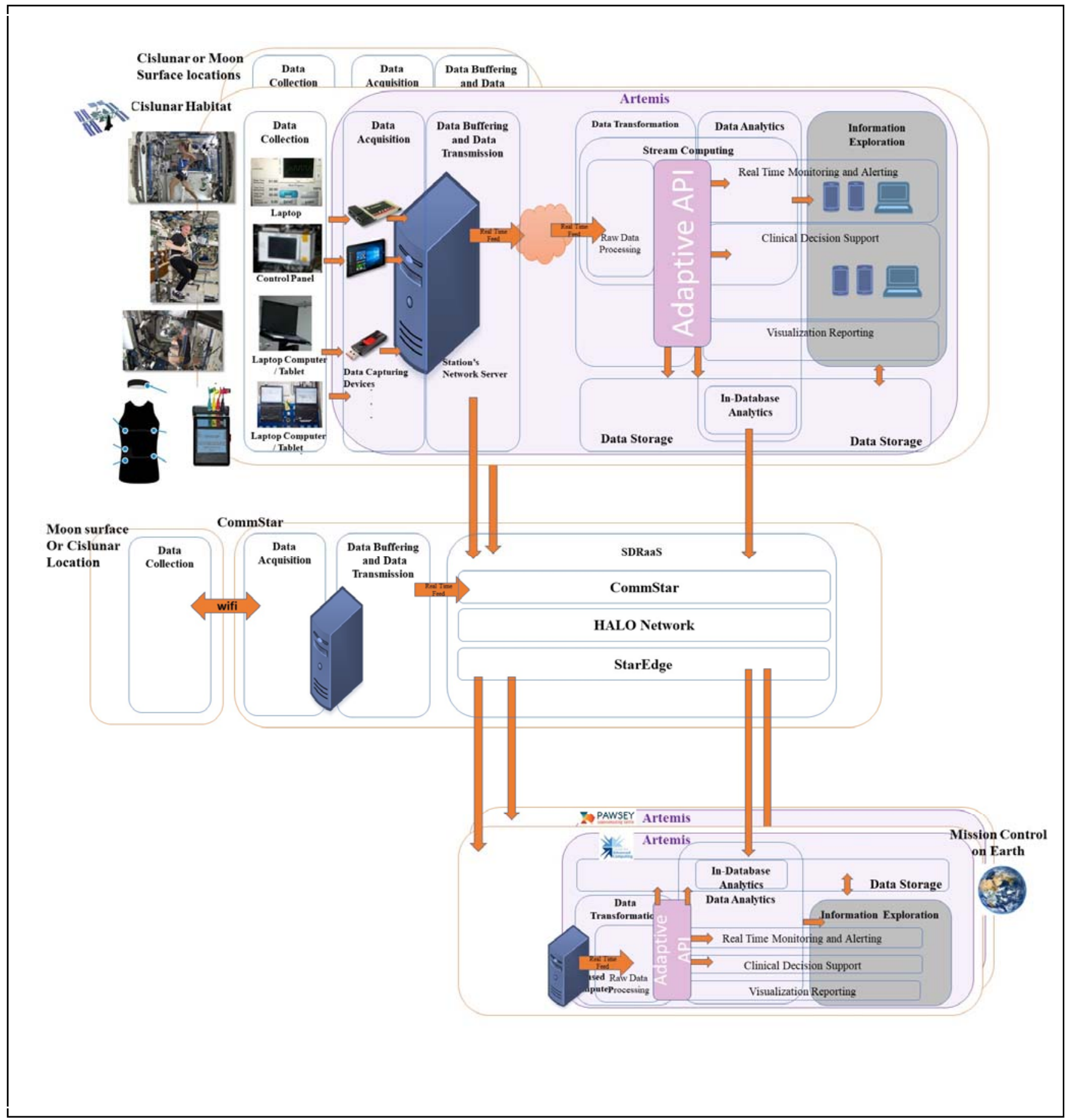

Figure 7. A Framework for SHAaaS

\section{Data Collection}

The SHAaaS enables astronaut health data to be collected via a range of IoT devices. In addition, countermeasure devices and their environment systems and sensors can also collect data that can be acquired and integrated into the SHAaaS. The SHAaas has the capacity to capture data while they are within their transportation and habitat spacecrafts, EVA suits, Moon based habitat together with Moon based habitat, working and transport environments.
Activity based tracking can be entered via manual web form entry or via other forms of passive surveillance such as location details and equipment usage details. As noted in [33] personal assessments and other astronaut checklists can be entered through a crew interface along with other medical history information. These can be transmitted using standardized formats such as HL7. 


\section{Data Acquisition}

All IoT data collection devices deployed for use in space should have the capacity to emit data tuples that should utilize standardized formats for the data streams and should be the subject of the establishment of a future set of standards. All IoT data collection devices should be enabled for wireless connectivity to enable data acquisition.

\section{Data Buffering and Data Transmission}

The Data Buffering component provides data message brokering. It manages the provision of messages for transmission through Data Transmission.

The Space Data Relay supports connectivity with devices that exist without a local Edge Computing component enabling Data Acquisition components to reach through the Space Data Relay to acquire data and then transmit and buffer as needed.

\section{Space Data Relay}

The Space Data Relay enables the relay of data between the Moon, cislunar and the Earth. It provides the network component to enable the cloud based provisioning of the SHAaaS. The Space Data Relay provides an additional layer of data buffering throughout the relay process.

\section{Data Transformation}

The Data Transformation component receives data and transforms it into formats published by the Adaptive API. In this way data standardization is enabled.

The Adaptive Application Program Interface (API), as expanded in Figure 8, ingests data from producers and publishes it for analytics within the Data Analytics component to consume. In addition, it forwards data to the Data Storage as a storage consumer.

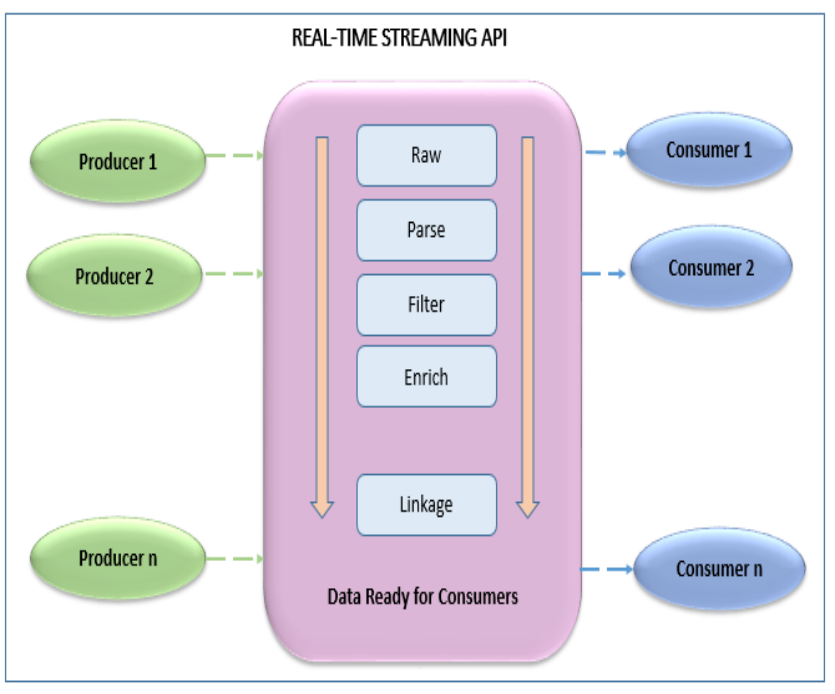

Figure 8. Real-Time Streaming Adaptive API [6]

\section{Data Analytics}

The data analytics component enables information, knowledge and wisdom to be derived from the acquired data. Data analytics can be performed in real-time as the data streams are forwarded from the Data Transformation component through the Adaptive API utilizing Stream Computing or can be performed in real-time and retrospectively using the data stored within the Data Storage layer.

Algorithms to enable the observation of the Bioastronautics Roadmap for adaptation would be deployed within the Data Analytics component. Additional algorithms to support general health and wellness observation would also be deployed.

\section{Information Exploration}

The information exploration layer provides access to the derived and raw data. It enables passive visualization and active visual analytics, where data can be dynamically explored visually. Information exploration can be tailored for different audiences such as the individual astronaut, CMO, the flight surgeon or equivalent and other roles.

\section{Data Storage}

The SHAaaS enables acquired data together with derived data from data analytics to be stored within the Data Storage component. The framework supports the use of separate data analytics tools and in database analytics tools to create new derived data.

\section{Prognostics and Health Management}

In addition to provisioning SHAaaS for astronaut health, wellness and adaptation monitoring, the same framework principle can be applied for prognostics and health management of equipment, spacecraft and structures applying the same principles to extend smart building and smart city approaches to smart space craft, smart space structure and smart space colony as a Service approaches.

\section{A Platform for Real-Time Space Health ANAlyTICS AS A SERVICE UTILIZING SDRAaS}

The framework outlined in the previous section is instantiated within a platform prototype design that utilizes Commstar-1 as part of the SDRaaS infrastructure to enable real-time SHAaaS.

Several of the architectural components have been tested within an Earth based analogue and others will be tested within Earth based analogues contexts prior to the commissioning of Commstar-1.

\section{Data Collection}

Currently the health monitoring devices on the ISS are the Cosmocard and the Bio-Monitor. As noted previously, these devices record data within a data logger while being worn and 
require uploading of data afterwards. Research using the Cosmocard device for data collection has been completed within the Luna 2015 analogue mission [1], [13] and also dry immersion experiments in 2016 [1], [15]. Testing with the Hexoskin product companion to the Bio-Monitor has been completed with firefighter students [34].

Future devices should enable output from the device in realtime to enable real-time data acquisition. Testing with this form of device will be part of future research.

\section{Data Acquisition}

Currently data acquisition from the Cosmocard and BioMonitor are via data download from the data loggers from each of the devices. In addition to the acquisition of data collected on the ISS, data acquisition from the Cosmocard device has been completed within the Luna 2015 analogue mission [1], [13] and also dry immersion experiments in 2016 [1], [15]. In these cases, data acquisition was performed post experiment. The Cosmocard data was acquired and preprocessed by the Cosmocard software. The Hexoskin data was acquired during studies with firefighter students [34] and provisioned to Artemis as flat files that were ingested by the API. The flat files were created using the Hexoskin cloud based software that connected with the data logger post experiment and produced the flat files.

\section{Data Buffering and Data Transmission}

Within the prototype instantiation of the SHAaaS, IBM InfoSphere streams can be used to manage the buffering and transmission of tuples within the data streams.

Within the current Artemis Cloud deployment [6], [7], Vines is used to manage data message queues, buffering and transmission. Vines utilizes Rabbit MQ. The JSON datainterchange format is utilized in the Artemis Cloud [6] and the Athena Cloud platform. Athena was created by McGregor to extend Artemis to incorporate activity and environmental data [35], [36].

Kafka can be used to support buffering and transmission functions and its potential for integration within Artemis Cloud has been discussed in [37].

\section{Space Data Relay}

The process of transmission of data is enabled through the SDRaaS. The SDRaaS is provisioned by CommStar, the HALO network and StarEdge as described previously in section 6 .

\section{Data Transformation}

The standardized API enables receipt of data from the devices providing health monitoring. Through related work to perform health and wellness monitoring of firefighter students while they train [35], [36] an API for Hexoskin, the terrestrial companion to the Bio-Monitor, has already been developed. Hexoskins as an analogue for the Bio-Monitor will be used for the initial prototype.

\section{Data Analytics}

IBM Infosphere Streams will be used to enable real-time stream computing based creation of new derived data from groupings of raw data and derived data within the Data Analytics component as has been demonstrated within the Artemis Cloud and Athena Cloud platforms [3], [6], [35]. This will support both real-time monitoring and alerting as well as clinical decision support.

For the in-database analytics, instances of the Artemis Cloud and Athena Cloud platform to date have utilized McGregor's temporal data mining approach, known as Service based Multidimensional Temporal Data Mining $\left(\mathrm{STDM}^{\mathrm{n}}{ }_{0}\right)$ [38], [3]. Various forms of AI based knowledge discovery can be utilized within the $\mathrm{STDM}_{0}^{\mathrm{n}}$ exploratory and explanatory components. $\mathrm{STDM}_{0}{ }_{0}$ will be utilized for in-database analytics support within SHAaaS.

\section{Information Exploration}

Within Artemis Cloud and Athena Cloud, the use of several off the shelf visualization tools have been tested and new visual analytics approaches have also been proposed [39]. Within this instantiation, Microsoft Power BI will be utilized similarly to its use in [35] to enable information exploration through both visualization and visual analytic functionality.

\section{Data Storage}

All Artemis, Artemis Cloud and Athena Cloud deployments to date use IBM DB2 for data storage [3], [6], [7] as the data is highly structured and the critical systems nature requires a high level of reliability and availability. In our initial prototype we will utilize IBM DB2 for the Data Storage layer.

\section{CONCLUSION AND FUTURE WORK}

This paper has presented a framework to enable real-time equipment monitoring for prognostics and health management and astronaut health monitoring through cloudbased Health Analytics as a Service utilizing space data relay. The framework is instantiated within a platform proposed as infrastructure utilizing a space data relay provisioned by Commstar and Laser Light.

A key benefit of this approach is its ability to monitor the astronauts' health and wellbeing onboard the spacecraft as well as enabling the equipment and astronaut's physiological data, and other clinical data, to be sent to Earth based Mission Control Center within more manageable latencies of seconds or minutes. This approach will provide a more viable alternative to autonomous only approaches for equipment and astronaut monitoring within and beyond LEO.

\section{ACKNOWLEDGEMENTS}

The author wishes to acknowledge the support of the Technical Experts Group at Laser Light Communications and CommStar Space Communications for providing details on 
their infrastructure. In particular, she would like to thank Bob Brumley, Glenn Colby, Rich Rosenthal and Steve Nichols.

\section{REFERENCES}

[1] O. Orlov, C. McGregor, R. Baevsky, A. Chernikova, A. Prysyazhnyuk, and V. Rusanov, "Perspective Use of the Technologies for Big Data Analysis in Manned Space Flights on the International Space Station," in 68th International Astronautical Congress (IAC 2017), 2017, p. in press.

[2] C. McGregor, "Big Data in Neonatal Intensive Care," Computer (Long. Beach. Calif)., vol. 46, no. 6, pp. 54-59, Jun. 2013.

[3] C. McGregor, "Big Data in Critical Care Using Artemis," in Signal Processing and Machine Learing for Biomedical Big Data, E. Sejdic and T. H. Falk, Eds. CRC Press, 2018.

[4] C. McGregor, "A Platform for Real-time Online Health Analytics during Spaceflight," in IEEE Aerospace Conference, 2013.

[5] C. McGregor, "A cloud computing framework for real-time rural and remote service of critical care," in Proceedings - IEEE Symposium on Computer-Based Medical Systems, 2011.

[6] C. Inibhunu, R. Jalali, I. Doyle, A. Gates, J. Madill, and C. McGregor, "Adaptive API for Real-Time Streaming Analytics as a Service," in 41st International Engineering in Medicine and Biology Conference, 2019, pp. 3472-3477.

[7] C. McGregor et al., "Health Analytics as a Service with Artemis Cloud: Service Availability," in Proceedings of the Annual International Conference of the IEEE Engineering in Medicine and Biology Society, EMBS, 2020, pp. 5644-5648.

[8] Commstar Space Communications, "Intention to Deploy Next-Generation Hybrid Data Relay Satellite Between the Earth and the Moon by 2023," 2020. [Online]. Available: https:/commstar.space/news/commstar-spacecommunications-launch-announcement/. [Accessed: 10-Oct-2020].

[9] A. Prysyazhnyuk and C. McGregor, "Space as an Extreme Environment," in Engineering and Medicine in Extreme Environments, T. Cibis and C. McGregor, Eds. Springer Nature, 2021.

[10] A. Prysyazhnyuk and C. Mcgregor, "Space as an Extreme Environment - Technical Considerations," in Engineering and Medicine in Extreme Environments, T. Cibis and C. McGregor, Eds. Springer Nature, 2021, pp. 1-20.
[11] C. McGregor, C. Catley, and A. James, "Variability analysis with analytics applied to physiological data streams from the neonatal intensive care unit," in Proceedings - IEEE Symposium on Computer-Based Medical Systems, 2012.

[12] R. M. Baevsky, A. G. Chernikova, I. I. Funtova, and J. Tank, "Assessment of individual adaptation to microgravity during long term space flight based on stepwise discriminant analysis of heart rate variability parameters," Acta Astronaut., vol. 69, no. 11, pp. 1148-1152, 2011.

[13] A. Prysyazhnyuk, C. McGregor, R. Baevsky, A. Chernikova, E. Luchitskaya, and V. Rusanov, "Big Data Analytics for Enhanced Clinical Decision Support Systems during Spaceflight," in IEEE Life Sciences Conference, 2017.

[14] A. E. Nicogossian, R. Baevsky, and N. G. House, "Chapter 4: In-Flight Medical Monitoring," in Space Biology and Medicine - Volume IV, Health, Performance, and Safety of Space Crews, American Institute of Aeronautics and Astronautics, Inc., 2004, pp. 61-85.

[15] A. Prysyazhnyuk, C. Mcgregor, E. Bersenev, and A. V. Slonov, "Investigation of adaptation mechanisms during five-day dry immersion utilizing big-data analytics," in 2018 IEEE Life Sciences Conference, LSC 2018, 2018, pp. 247-250.

[16] Carre Technologies inc (Hexoskin), "The BioMonitor to Launch to the International Space Station," 2020. [Online]. Available: https://www.hexoskin.com/blogs/news/bio-monitorastroskin-to-launch-to-the-international-spacestation. [Accessed: 16-Oct-2020].

[17] H. J. Kramer, "ISS Utilization: Bio-Monitor and BioAnalyzer." [Online]. Available: https://directory.eoportal.org/web/eoportal/satellitemissions/i/iss-bio-monitor-analyzer. [Accessed: 16Oct-2020].

[18] Canadian Space Agency, "Investigating Spaceflight Health Risks on the ISS (LSRS AO 2018)," 2018. [Online]. Available: https:/Www.asccsa.gc.ca/eng/funding-programs/fundingopportunities/ao/2018-lsrs.asp\#appendix-a-bio-m. [Accessed: 16-Oct-2020].

[19] D. J. Newman, "Life in extreme environments: how will humans perform on Mars?," Gravitational and space biology bulletin: publication of the American Society for Gravitational and Space Biology, vol. 13, no. 2. 2000 .

[20] A. Rafiq, "Space crew health monitoring," in Wireless Sensor and Mobile Ad-Hoc Networks 
Vehicular and Space Applications, Springer New York, 2015, pp. 149-160.

[21] C. Panteli, A. Kylili, and P. A. Fokaides, "Building information modelling applications in smart buildings: From design to commissioning and beyond A critical review," J. Clean. Prod., vol. 265, 2020.

[22] C. McGregor, C. Catley, A. James, and J. Padbury, "Next Generation Neonatal Health Informatics with Artemis," in User Centred Networked Health Care, IOS Press, 2011, pp. 115-119.

[23] C. McGregor, "A platform for real-time online health analytics during spaceflight," in IEEE Aerospace Conference Proceedings, 2013.

[24] J. Yeung and C. McGregor, "Analyzing Countermeasure Effectiveness Utilizing Big Data Analytics for Space Medicine Decision Support: A Case Study Jennifer Yeung," in 70th International Astronautical Congress (IAC), 2019, no. October.

[25] Canadian Space Agency, "ACMS SMDSS RFI (9F008-150293/A)," 2016. [Online]. Available: https://buyandsell.gc.ca/cds/public/2016/07/21/ce21 b3d9c1ab2b55122a526b38eea330/ABES.PROD.P W_MTB.B545.E13964.EBSU000.PDF.

[26] (NASA) National Aeronautics and Space Administration, "Space Network (SN)," Exploration and Space Communications. [Online]. Available: https://esc.gsfc.nasa.gov/projects/SN?tab=sn fleet operations. [Accessed: 08-Oct-2020].

[27] (NASA) National Aeronautics and Space Administration, "Data Rate Increase on the International Space Station Supports Future Exploration," Space Communications, 2019. [Online]. Available: https://www.nasa.gov/feature/goddard/2019/datarate-increase-on-the-international-space-stationsupports-future-exploration. [Accessed: 08-Oct2020].

[28] CommStar Space Communications, "CommStar Briefing Slides," 2020.

[29] Atlas Space Operations and Laser Light, "Relay Services Architecture Description Redacted Rev $1.1, " 2019$.

[30] Laser Light Communications, "Laser Light Advantage," 2020. [Online]. Available: https://www.laserlightcomms.com/. [Accessed: 16Oct-2020].

[31] Atlas Space Operations and Laser Light Communications, "Relay Services Concept of Operations," 2019.
[32] I. Sittón-Candanedo, R. S. Alonso, Ó. García, L. Muñoz, and S. Rodríguez-González, "Edge computing, iot and social computing in smart energy scenarios," Sensors (Switzerland), vol. 19, no. 15, 2019.

[33] A. Prysyazhnyuk and C. McGregor, "A Wholistic Approach to Assessment of Adaptation and Resilience During Spaceflight," in 71st International Astronautical Congress (IAC), The CyberSpace Edition, 2020, no. October, pp. 12-14.

[34] C. McGregor, F. M. Williams-Bell, and B. Bonnis, "Using Streaming Big Data for Resilience Assessment within Pre-service Firefighter Extreme Condition Training," in The 9th annual Military and Veteran Health Research Forum (CIMVHR), 2018, p. 29.

[35] C. McGregor, A. Prysyazhnyuk, and B. Bonnis, "Using Big Data for Pre-deployment Acclimation Strategies Incorporating Resilience Assessment and Development for Extreme Heat Climatic Conditions Using Controlled Climate Chambers," Oshawa, Canada, 2019.

[36] C. McGregor, F. M. Williams-Bell, and B. Bonnis, "Extreme Climate Pre-deployment Acclimation Resilience Assessment Using Climate Chambers and Big Data," in 10th Annual Military and Veteran Health Research Forum, 2019, p. 87.

[37] A. Novakovic, A. H. Marshall, and C. McGregor, "Introducing a Conceptual Framework for Architecting Healthcare 4.0 Systems," in Transactions on Computational Science \& Computational Intelligence (Advances in Computer Vision and Computational Biology), Springer Nature, 2020

[38] C. McGregor, "System, Method and Computer Program For Multi-Dimensional Temporal Data Mining,” WO2011009211 A1, 27-Jan-2011.

[39] R. Kamaleswaran, C. Collins, A. James, and C. McGregor, "PhysioEx: Visual Analysis of Physiological Event Streams," Comput. Graph. Forum, vol. 35, no. 3, 2016.

\section{BIOGRAPHY}

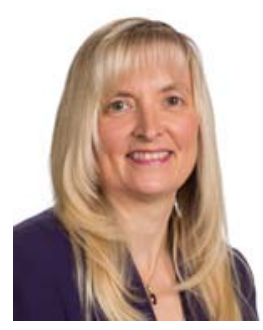

Carolyn McGregor AM is the Research Chair in Artificial Intelligence for Health and Wellness and a two-time Canada Research Chair in Health Informatics at Ontario Tech University. She is the Founding Director of the Joint Research Centre in Artificial Intelligence for Health and Wellness between Ontario Tech 
University and the University of Technology Sydney. Dr McGregor AM has led pioneering research in Big Data analytics, artificial intelligence, deep learning, internet of things, temporal data mining and cloud computing. She now progresses this research within the context of critical care medicine, precision public health, mental health, astronaut health and military and civilian tactical training. 